\title{
DENDROPHTHORA COSTARICENSIS (LORANTHACEAE), UN NUEVO REGISTRO PARA LA FLORA DE MEXICO
}

\author{
Miguel Chazaro Basanez \\ Departamento de Investigación Científica y \\ Superación Académica (D.I.C.S.A.) \\ Universidad de Guadalajara, Guadalajara, Jalisco, México \\ Hector Oliva Rivera \\ Facultad de Ciencias Biológicas, \\ Universidad Veracruzana \\ Apartado postal \#177, Córdoba, Veracruz, México
}

\begin{abstract}
RESUMEN
Dendrophthora costaricensis Urban se registra por primera vez para México; se encontró en las laderas de barlovento del Cofre de Perote en el centro de Veracruz, México.
\end{abstract}

\section{ABSTRACT}

Dendrophthora costaricensis Urban, is recorded from Mexico for the first time. It was found on the seaward slopes of Cofre de Perote volcano in central Veracruz, Mexico.

\section{INTRODUCCION Y ANTECEDENTES}

Las Loranthaceae sensu lato o Loranthaceae, Viscaceae y Eremolepidaceae sensu stricto, constituyen una familia de plantas parásitas de los troncos y/o ramas de árboles 0 arbustos, tanto de Gimnospermas (confferas), como de Dicotiledóneas y muy raramente de Monocotiledóneas.

En nuestro país existen 9 géneros: Antidaphne Poepp. \& Endl., Orycthanthus Eichler, Phthirusa Mart., Arceuthobium Bieb., Phoradendron Nuttall, Dendrophthora Eichler, Cladocolea van Tieghem, Struthanthus Mart. y Psittacanthus Mart.

Las dos especies de Phrygilanthus Eichler, citadas por Standley (1920-1926) en su obra sobre árboles y arbustos de México, fueron reacomodadas dentro de Psittacanthus; Phrygilanthus palmeri (Watson) Engl., pasó a ser Psittacanthus palmeri (Watson) Barlow \& Wiens y, Phrygilanthus sonorae (Watson) Standl. a P. sonorae (Watson) Kuijt.

Cabe aquí señalar el hecho de que muchas especies de Loranthaceae americanas descritas por taxónomos europeos o norteamericanos durante el siglo XVIII y XIX fueron inicialmente colocadas en Viscum y Loranthus, géneros que hoy se consideran exclusivos del viejo mundo.

Dendrophthora es un género que, de acuerdo con Kuijt (1961), está representado por 53 especies, todas ellas de América tropical.

En las islas del Caribe, se conoce de Cuba, Española, Jamaica, Puerto Rico, Santa Lucía y Martinica, siendo el oriente de Cuba y Española donde se encuentra el mayor número de especies (Kuijt,1961). 
En América continental se distribuye también, de acuerdo con el mismo autor desde el sur de México hasta Bolivia, siguiendo las cadenas montañosas.

Por otro lado, es importante enfatizar la similitud morfológica de algunas especies de Phoradendron con las de Dendrophthora, pues la única diferencia entre estos dos géneros reside en el número de lóculos de las anteras (dos en Phoradendron y uno en Dendrophthora). Tal hecho ha complicado la taxonomía de ambos géneros, causando con ello muchos problemas y errores de identificación en el pasado y aún en la actualidad, ya que las flores en ambos géneros son diminutas y el carácter que las separa es difícil de observar.

El primer registro del género Dendrophthora publicado para México, aparece en la monumental obra de Standley (1920-1926), donde se cita $D$. pedicellata van Tieghem para la localidad de Mesa Chica.

Sin embargo, Kuijt en su monografía del género no menciona tal especie. Guiándonos por la localidad que da Standley (Mesa Chica; conocemos una ranchería con tal nombre entre San José Acateno, Pue. y Papantla, Ver.), así como por la descripción que señala la presencia de frutos pedicelados, y al no encontrar ejemplares de respaldo en los principales herbarios mexicanos para $D$. pedicellata, dedujimos que debe tratarse muy probablemente de Phoradendron wawrae Trelease, pues nos consta que tal especie crece en Mesa Chica, Veracruz y tiene los frutos pedicelados, quedando por consiguiente con mucha duda este primer registro.

A pesar de las cuantiosas colectas hechas por el Dr. Eizi Matuda en la región del Soconusco, Chiapas, este autor (Matuda, 1950) no registra la presencia de Dendrophthora.

Kuijt (1961) menciona una sola especie para México, D. mexicana, descrita por él mismo de ejemplares colectados por Quarles Van Ufford (No. 263) en Niquihuil (Chiapas), siendo por lo tanto éste el primer registro válido para México, además de representar la localidad más septentrional del género conocida para esa fecha.

Más recientemente en su listado florístico de Chiapas, además de $D$. mexicana Kuijt, Breedlove (1986) adiciona a $D$. guatemalensis Standley como un nuevo registro para la flora de México.

\section{RESULTADOS}

Al realizar colectas botánicas en el centro de Veracruz en 1981-1982 y en los años ulteriores en forma esporádica, nos cruzamos con un raro y vistoso "muérdago", el cual en otra publicación nuestra (Cházaro y Oliva, 1987) fue identificado temporalmente como Phoradendron aff. oliverianum Trelease, mencionando que podría tratarse de una especie inédita.

Ejemplares colectados en Presa de Mazatepec, Puebla, $900 \mathrm{~m}$ s.n.m., 18 de abril de 1973, L. Wolfgang Boege 2784 (ENCB); El Mirador, Mpio. de Totutla, Ver., $1000 \mathrm{~m}$ s.n.m., 23 de junio de 1981 (frutos) F. Ventura Aburto 18652 (ENCB, XAL) (esta segunda es precisamente la localidad tipo de $P$. oliverianum); Atehuiztita, $6 \mathrm{~km}$ al SE de Zapotitán, brecha a Zacapoaxtla, mpio. Zapotitán de Méndez, Puebla, 700 m s.n.m., 4 de julio 1987 (frutos) Pedro Tenorio L., A. Campos V., G. Gómez A. 13925 (MEXU, MICH) y determinados correctamente como $P$. oliverianum, nos convencieron que nuestro espé- 
cimen de Buenavista, Mpio. de Xico, corresponde a otro taxon, como ya lo habíamos sospechado. Dado que Phoradendron oliverianum Trel. es una planta rara, incluimos a continuación un dibujo de esta especie (Fig. 1).

En 1985 gracias a la cortesía del Dr. William C. Burger, llegó a nuestras manos la publicación sobre las Lorantáceas de Costa Rica (Burger y Kuijt, 1983) y alli pudimos darnos cuenta del parecido asombroso entre nuestro Phoradendron aff. oliverianum y Dendrophthora costaricensis, pero el hecho de estar el centro de Veracruz tan lejos del área de distribución conocida de este último, nos hizo titubear en asignar una determinación definitiva.

Años después, trabajando con las Lorantáceas del Herbario Nacional de México (MEXU), encontramos especímenes de intercambio de $D$. costaricensis colectados, uno en Costa Rica y otro en Panamá. Después de un minucioso escrutinio, no nos quedó la menor duda de que nuestra planta de Buenavista pertenece a la mencionada especie.

A continuación se presenta una descripción basada en los ejemplares colectados en Veracruz.

Dendrophthora costaricensis Urban. Ber. Deutsch. Bot. Gesell. 14: 285. 1896.

Hierba perenne de 20 a $40 \mathrm{~cm}$ de longitud con los entrenudos basales teretes, los entrenudos basales de $3-4 \mathrm{~cm}$ de largo, los subsiguientes se van acortando progresivamente hacia la parte distal de las ramillas, ramas opuestas, con un par de catafilas situadas a 5-8 mm de la axila; hojas opuestas, carnosas, 3-5 nervadas desde la base, pero los nervios no son muy manifiestos, con superficie ampulosa en ambas caras, lámina de la hoja espatulada, con el ápice obtuso a redondeado, la base decurrente, margen ligeramente ondulado en los especímenes secos (no así en fresco), con una línea amarillenta visible a contraluz, de (3) $4-5.5(6.5) \mathrm{cm}$ de largo, por 1.5 a $2.5 \mathrm{~cm}$ de ancho en hojas maduras; inflorescencia en forma de espigas axilares, con 2 a 3 entrenudos o segmentos fértiles por espiga, flores 8-5 por segmento; fruto blanco translúcido, de forma esférica (Fig. 2).

Especímenes examinados: Panamá. Prov. Chiriquí, $6 \mathrm{~km}$ east of Cerro Punta, Paso Respingo area, 7800 feet, 12 febrero 1980, Barry Hammel 1507 (MEXU, MO). Costa Rica. Cerro de la Muerte, 3000 m s.n.m., 7 de octubre 1978, Antonio Thomas 683 (F, MEXU). Honduras. Dpto. La Paz, bosque nebuloso de montaña verde, aldea Las Marías, Cordillera Guajiquiro, 2100 m s.n.m, 23 de mayo 1964, Antonio Molina 14048 (F). El Salvador. Dpto. Santa Ana, moist cloud forest on cordillera Miramundo, Mountain of Montecristo, El Trifino, 2000-2200 m s.n.m., 27-31 enero de 1966, Antonio Molina, W.C. Burger \& B. Wallenta 16800 (F). Guatemala. Dpto. Zacapa, between Loma el Picacho and Cerro de Monos, 2000-2600 m s.n.m., 16 enero de 1942, J. A. Steyermark 42816 (F). Dpto. Chiquimula, middle slopes of Montaña Norte to El Juncal, on Cerro Brujo, SE of Concepción de las Minas, 1700-2000 m s.n.m., 2 noviembre 1939, J.A. Steyermark 30993 (F). Dpto. San Marcos, Barranco Eminencia, above San Rafael Pie de la Cuesta, 2100-2400 m s.n.m., 14-15 marzo 1939, P.C. Standley 68469 (F). México. Veracruz, Mpio. Xico, barranca entre Tlibaya y Coxmatla, 2100 m s.n.m., 2 abril 1983 (flores), M. Cházaro B. y Luis Robles H. 2687 (LEA, WIS). Mpio. de Xico, de Xico a Corral de Rajas, 1800 m s.n.m., 7 agosto 


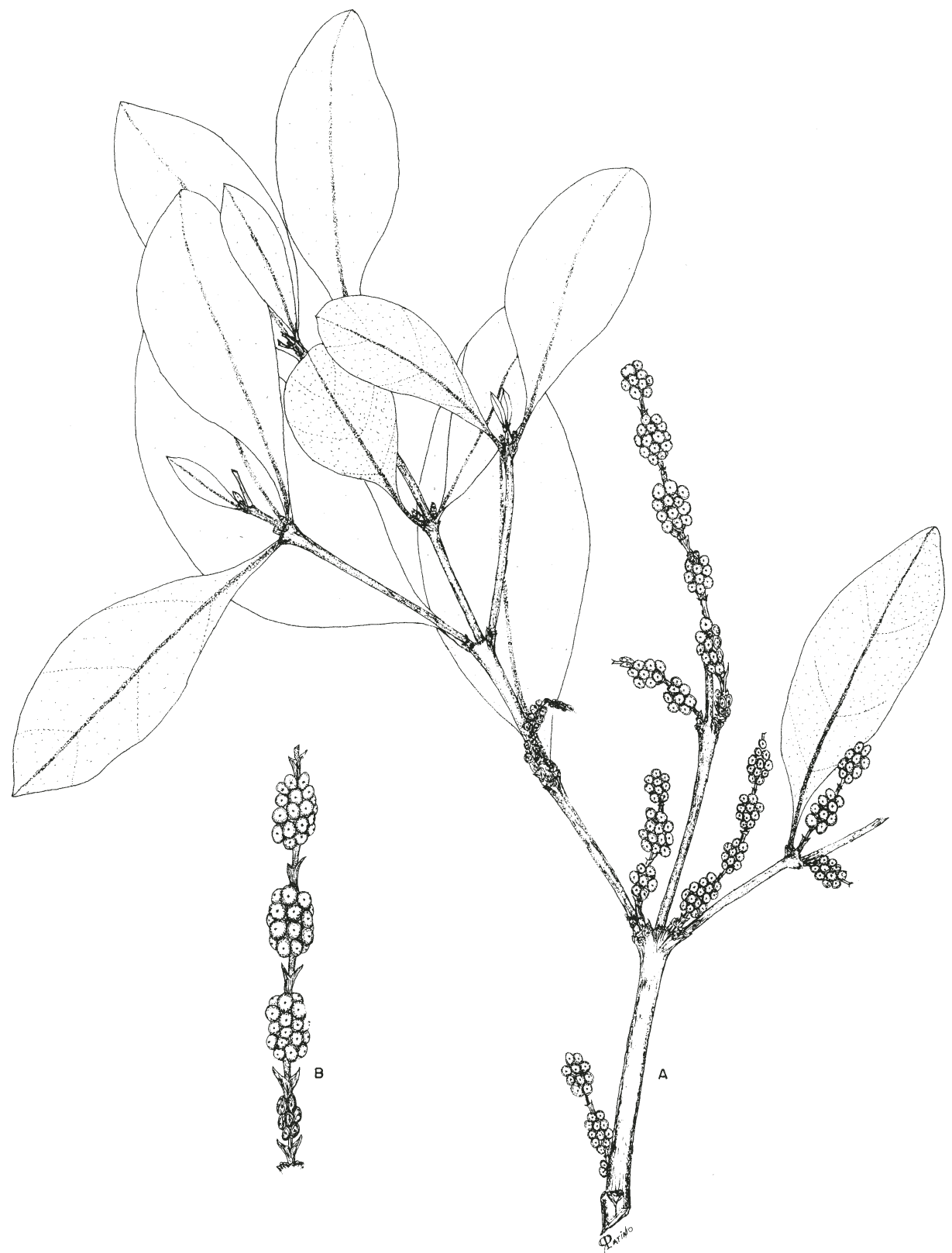

Fig. 1. Phoradendron oliverianum Trel. A. Rama con infrutescencias; B. Infrutescencias (P. Tenorio L. et al. 13925, MICH Y MEXU). 
Cházaro y Oliva: Dendrophthora costaricensis Nuevo Registro para México

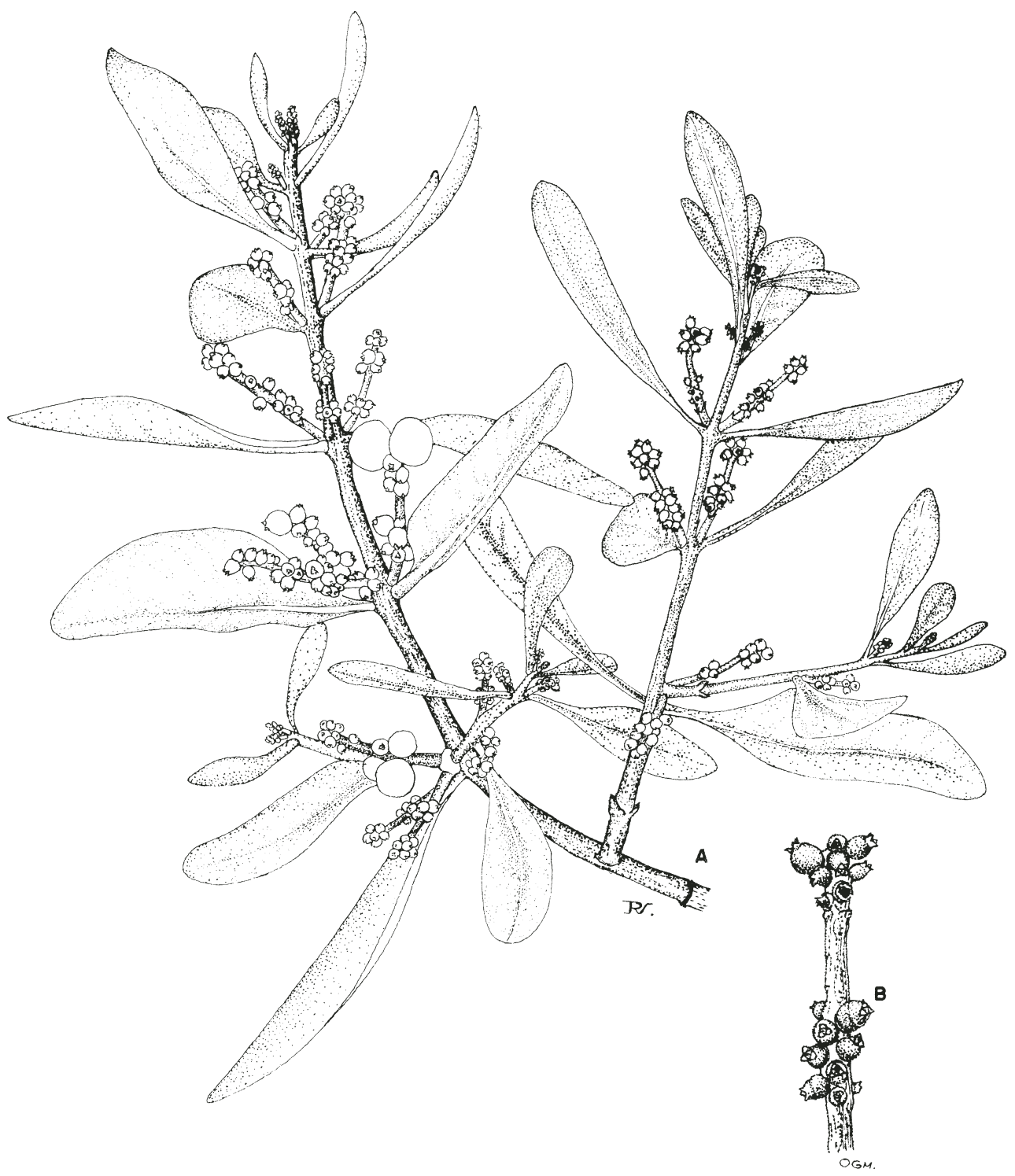

Fig. 2. Dendrophthora costaricensis Urban (M. Cházaroy H. Oliva 5253, IBUG). A. Ramilla con frutos jóvenes y maduros; B. Espiga femenina, con frutos muy jóvenes. 
1986 (flores y frutos), M. Cházaro B. y Luis Robles H. 3836 (IBUG, LEA, WIS, XAL). Mpio. de Xico, cerca de Buenavista, 2100 m s.n.m., 12 de octubre 1987 (frutos) M. Cházaro B. y Patricia Hdez. de Ch. 5062 (F, IBUG). Mpio. de Xico, cerca de Buenavista, $2200 \mathrm{~m}$ s.n.m., 25 de diciembre 1987 (flores y frutos), M. Cházaro B. y H. Oliva R. 5253 (ANSM, CAS, CHAPA, IEB, LEA, MEXU, WIS, XAL).

D. costaricencis fue originalmente descrita de ejemplares colectados en el Volcán Irazú en Costa Rica. Rizzini (1960) la menciona para dos localidades de la provincia de Chiriquí, Panamá. En 1961 Kuijt la registra de Costa Rica y Panamá, pero en 1963 ya se conocía su existencia también en El Salvador y Guatemala (Kuijt, 1963). Por ejemplares de Herbario depositados en (F) sabemos que también prospera en Honduras, al igual que en Colombia (J. Kuijt, comunicación personal, 1990).

De Guatemala aparentemente el área se interrumpe hasta Veracruz, pues no existen ejemplares de Chiapas ni tampoco de Oaxaca.

De existir la especie en estas dos últimas entidades federativas tendrá que buscarse en algún lugar con características ecológicas similares a las de Buenavista, donde este muérdago se encontró en las laderas húmedas y neblinosas del Cofre de Perote, cubiertas por un bosque de niebla (sensu Cházaro, 1982), o evergreen cloud forest (sensu Breedlove, 1973), compuesto por Drimys, Weinmannia, Symplocos, Styrax, "Cleyera, Quercus, Zanthoxylum, Ternstroemia, Osmantus, Vaccinium leucanthum, Turpinia occidentalis, etc. Miconia se halla dominando el estrato arbustivo, siendo esta Melastomataceae el hospedero principal de $D$. costaricensis, aunque en dos ocasiones la encontramos también sobre Rondeletia sp.

Este novedoso hallazgo de Dendrophthora costaricensis en un área restringida de Veracruz, además de representar la tercera especie del género para México, amplía el límite septentrional de distribución de Dendrophthora por más de $500 \mathrm{~km}$, pues la población conocida más próxima se localiza cerca de Tuxtla Gutiérrez (Cañón del Sumidero), donde crece D. guatemalensis (ver Fig. 3).

Dado que en Veracruz sólo conocemos esta planta de un área restringida (cerca de Buenavista y entre Coxmatla y Tlibaya), donde es abundante localmente, se podría postular la hipótesis de que se trata de una introducción reciente, efectuada a larga distancia por aves.

\section{AGRADECIMIENTOS}

Al Biól. Luis Robles Hernández del Laboratorio Natural Las Joyas, Universidad de Guadalajara, El Grullo, Jalisco, quien nos acompaño en los viajes de colecta. Al Dr. William C. Burger, del Museo Field de Historia Natural de Chicago, Illinois, por el envío de Fieldiana Botany 13 (Flora Costaricensis), así como por permitir la revisión de los exsiccata de Loranthaceae depositados en el Herbario F. Al Dr. Frank G. Hawksworth, del Servicio Forestal del Dpto. de Agricultura de los Estados Unidos, en Fort Collins, Colorado, por la revisión al manuscrito. Al Dr. Job Kuijt por la revisión del manuscrito e información personal proporcionada.

A la Dra. Patricia Dávila A., jefe del Herbario Nacional de México (MEXU), por el préstamo de los exsiccata de Dendrophthora costaricensis procedentes de Costa Rica y 
Cházaro y Oliva: Dendrophthora costaricensis Nuevo Registro para México

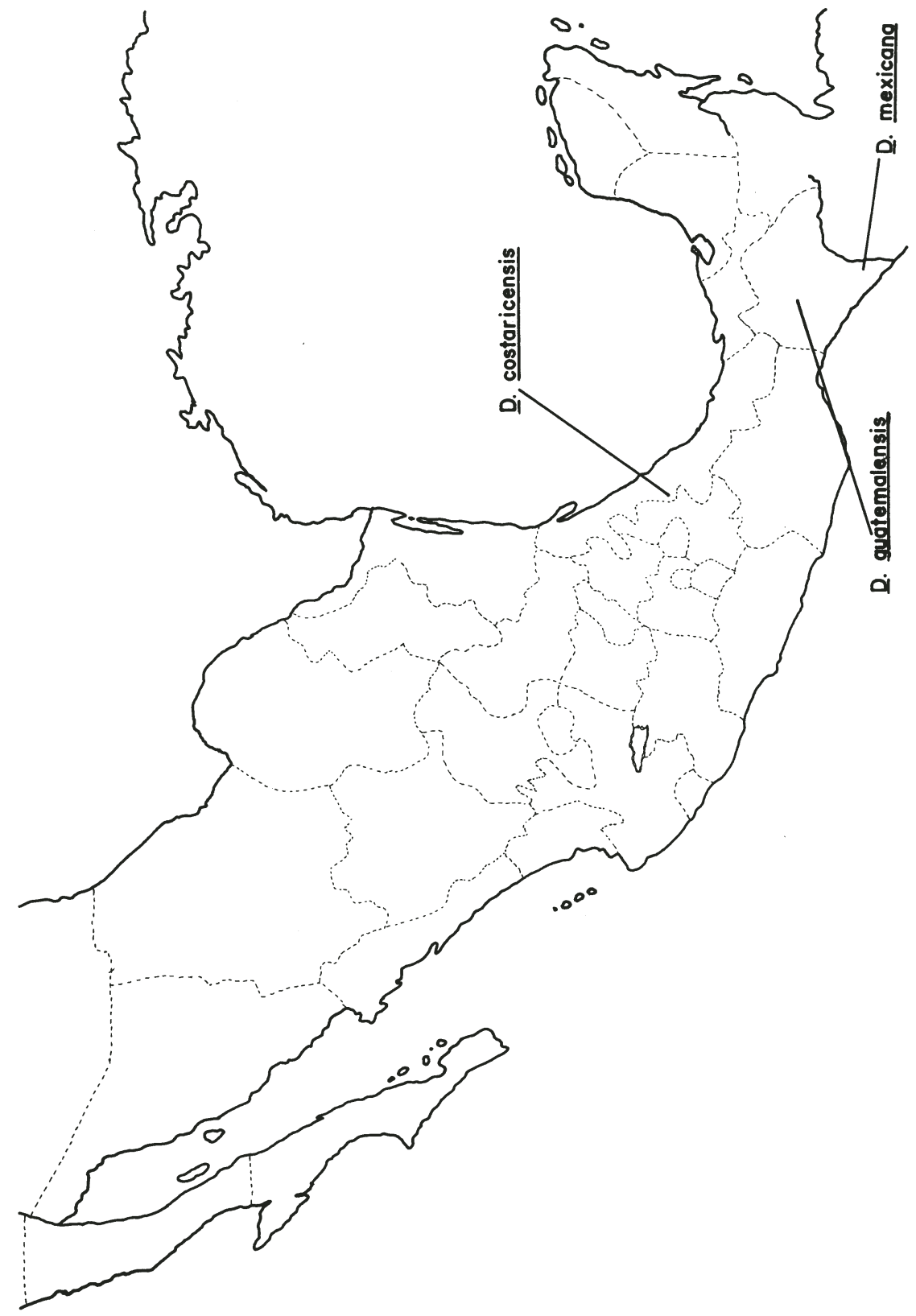

Fig. 3. Distribución del género Dendrophthora en México. 
Panamá. A María del Refugio Vázquez Velasco, del Instituto de Botánica, Universidad de Guadalajara y a Rosa María Patiño Beltrán, del Instituto de Geografía y Estadística, Universidad de Guadalajara, por los dibujos.

M. Cházaro B. desea agradecer de forma especial, la gran ayuda prestada por nuestro profesor el Dr. Hugh $\mathrm{H}$. Iltis, a través de quien hemos obtenido ayuda financiera del Herbario de la Universidad de Wisconsin, Madison, que nos hizo posible realizar los viajes de campo. Finalmente a mi querida esposa Patricia Hernández de Cházaro, quien además de realizar el trabajo mecanográfico, ha sido una hábil y valiosa compañía en el campo, durante más de 10 años.

\section{LITERATURA CITADA}

Breedlove, D. E. 1973. The phytogeography and vegetation of Chiapas (México). In: Graham, A. Vegetation and Vegetation history of northern Latin America. Elsevier Publishing Company. Amsterdam.

Breedlove, D. E. 1986. Loranthaceae. Listados florísticos de México. V. Flora de Chiapas. Instituto de Biología, Universidad Nacional Autónoma de México. México, D.F. 187 pp.

Burger, W. C. y J. Kuijt. 1983. Loranthaceae, In: Burger, W. C. Flora Costaricensis. Fieldiana: Botany 13: 29-79.

Cházaro B., M. 1982. Pisos altitudinales de vegetación en el Centro de Veracruz y zona límitrofe de Puebla. Manuscrito inédito. Xalapa, Veracruz. 29 pp.

Cházaro B., M. y H. Oliva R. 1987. Loranthaceae del Centro de Veracruz y zona limítrofe de Puebla I, II. Cact. y Suc. Mex. 32(4): 78-86.

Kuijt, J. 1961. A revision of Dendrophthora (Loranthaceae). Wentia 6: 1-145.

Kuijt, J. 1963. Dendrophthora: additions and changes. Acta Bot. Neerlandica 12: 521-524.

Matuda, E. 1950. A contribution to our knowledge of the wild and cultivated flora of Chiapas. 1. Districts Soconusco and Mariscal. Amer. Midl. Natur. 44(3): 513-616.

Rizzini, C. T. 1960. Loranthaceae. In: Woodson, R. E., Jr. \& R. W. Schery. Flora of Panama. Ann. Missouri Bot. Gard. 47: 263-290.

Standley, C. P. 1920-1926. Trees and shrubs of Mexico. Contrib. U.S. Nat. Herb. 23: 227-235. 